Bull. Korean Math. Soc. 51 (2014), No. 2, pp. 373-386

http://dx.doi.org/10.4134/BKMS.2014.51.2.373

\title{
COHEN-MACAULAY MODULES OVER NOETHERIAN LOCAL RINGS
}

\author{
KAMAL BAHMANPOUR
}

\begin{abstract}
Let $(R, \mathfrak{m})$ be a commutative Noetherian local ring. In this paper we show that a finitely generated $R$-module $M$ of dimension $d$ is Cohen-Macaulay if and only if there exists a proper ideal $I$ of $R$ such that $\operatorname{depth}\left(M / I^{n} M\right)=d$ for $n \gg 0$. Also we show that, if $\operatorname{dim}(R)=d$ and $I_{1} \subset \cdots \subset I_{n}$ is a chain of ideals of $R$ such that $R / I_{k}$ is maximal Cohen-Macaulay for all $k$, then $n \leq \ell_{R}\left(R /\left(a_{1}, \ldots, a_{d}\right) R\right)$ for every system of parameters $a_{1}, \ldots, a_{d}$ of $R$. Also, in the case where $\operatorname{dim}(R)=2$, we prove that the ideal transform $D_{\mathfrak{m}}(\mathrm{R} / \mathfrak{p})$ is minimax balanced big Cohen-Macaulay, for every $\mathfrak{p} \in \operatorname{Assh}_{R}(R)$, and we give some equivalent conditions for this ideal transform being maximal Cohen-Macaulay.
\end{abstract}

\section{Introduction}

Let $R$ denote a commutative Noetherian ring (with identity), $I$ an ideal of $R$, and $M$ a finitely generated $R$-module. In [11] L. J. Ratliff, Jr., conjectured about the asymptotic behavior of $\operatorname{Ass}_{R}\left(M / J^{n} M\right)$ when $R$ is a Noetherian domain. Subsequently, M. Brodmann in [1] showed that if $R$ is a Noetherian ring, then the sets $\operatorname{Ass}_{R}\left(M / J^{n} M\right)$ and $\operatorname{Ass}_{R}\left(J^{n} M / J^{n+1} M\right)$ are ultimately constant for large $n$. Also, based on this result, in [2] he showed that if $R$ is a Noetherian ring, then the integers $\operatorname{depth}\left(I, M / J^{n} M\right)$ and $\operatorname{depth}\left(I, J^{n} M / J^{n+1} M\right)$ take constant values for large $n$. In particular, the integers $\operatorname{depth}_{R}\left(M / J^{n} M\right)$ and $\operatorname{depth}_{R}\left(J^{n} M / J^{n+1} M\right)$ take constant values for large $n$, whenever $(R, \mathfrak{m})$ is a Noetherian local ring. In Section 2 of this paper we show that if there exists an ideal $J$ of $R$ in which the constant value of $\operatorname{depth}_{R}\left(M / J^{n} M\right)$ is equal to $\operatorname{dim}(M)$, then $M$ is Cohen-Macaulay. More precisely, we shall prove the following characterization of Cohen-Macaulay modules over Noetherian local rings:

Received June 17, 2012; Revised August 27, 2013.

2010 Mathematics Subject Classification. 13D45, 14B15, $13 \mathrm{E} 05$.

Key words and phrases. balanced big Cohen-Macaulay modules, Cohen-Macaulay modules, local cohomology modules, quasi regular sequences.

This research of the author was in part supported by a grant from IPM (No. 91130022). 
Theorem 1.1. Let $(R, \mathfrak{m})$ be a Noetherian local ring and $M$ be a non-zero finitely generated $R$-module of dimension $d$. Then the following conditions are equivalent:

(i) $M$ is Cohen-Macaulay.

(ii) There exists a proper ideal $I$ of $R$ such that $\operatorname{depth}_{R}\left(M / I^{n} M\right)=d$ for all $n \gg 0$.

Recall that a finitely generated module $M$ over a Noetherian local ring $(R, \mathfrak{m})$ is said to be maximal Cohen-Macaulay if $\operatorname{depth}_{R}(M)=\operatorname{dim}(M)=$ $\operatorname{dim}(R)$. Note that if $M$ is a maximal Cohen-Macaulay, then any system of parameters of $R$ is also a system of parameters of $M$ and therefore it is an $M$-regular sequence. In Section 2 we shall prove the following results about Cohen-Macaulay modules:

Theorem 1.2. Let $(R, \mathfrak{m})$ be a Noetherian local ring of dimension $d \geq 1$ and

$$
I_{1} \subset I_{2} \subset \cdots \subset I_{n-1} \subset I_{n}
$$

be a chain of ideals of $R$ such that for each $1 \leq k \leq n$, the $R$-module $R / I_{k}$ is maximal Cohen-Macaulay. Then for each system of parameters $x_{1}, \ldots, x_{d}$ of $R$, we have

$$
n \leq \ell_{R}\left(R /\left(x_{1}, \ldots, x_{d}\right)\right),
$$

where $\ell_{R}\left(R /\left(x_{1}, \ldots, x_{d}\right)\right)$ denotes the length of the $R$-module $R /\left(x_{1}, \ldots, x_{d}\right)$.

Theorem 1.3. Let $(R, \mathfrak{m})$ be a Noetherian local ring of dimension $d \geq 1$ and $I$ be an ideal of $R$ such that $\operatorname{dim}(R / I)=n \geq 1$. Let $x_{1}, \ldots, x_{n}$ be a system of parameters for $R / I$. If $R / I$ is a Cohen-Macaulay module and the ideal $Q:=I+R x_{1}+\cdots+R x_{n}$ is a parameter ideal of $R$, then there are elements $a_{1}, \ldots, a_{d-n} \in I$ such that $I=R a_{1}+\cdots+R a_{d-n}$ and $x_{1}, \ldots, x_{n}, a_{1}, \ldots, a_{d-n}$ is a system of parameters for $R$. In particular, if $R$ is Cohen-Macaulay, then $I$ is generated by an $R$-sequence.

For proving these results we shall use the basic properties of regular and quasi regular sequences. Recall that if $R$ is a commutative ring with identity, $M$ is an $R$-module, $X_{1}, \ldots, X_{n}, n$ indeterminants and $Z_{R}(N)$ denotes to the set of all zero-divisors of $N$ in $R$, for each non-zero $R$-module $N$, then an ordered sequence $a_{1}, \ldots, a_{n} \in R$ is said to be an $M$-regular sequence if for all $1 \leq i \leq n, a_{i} \notin Z_{R}\left(M /\left(a_{1}, \ldots, a_{i-1}\right) M\right)$ and $\left(a_{1}, \ldots, a_{n}\right) M \neq M$. Also a sequence (not necessary ordered) $a_{1}, \ldots, a_{n} \in R$ is said to be an $M$-quasi-regular sequence if $\left(a_{1}, \ldots, a_{n}\right) M \neq M$ and for each integer $k$ and each homogeneous polynomial $P\left(X_{1}, \ldots, X_{n}\right) \in M\left[X_{1}, \ldots, X_{n}\right]$ of degree $k$, $P\left(a_{1}, \ldots, a_{n}\right) \in\left(a_{1}, \ldots, a_{n}\right)^{k+1} M$ implies that all the coefficients of $P$ are in $\left(a_{1}, \ldots, a_{n}\right) M$.

In the sequel let $(R, \mathfrak{m})$ be a Noetherian local ring of dimension $d \geq 1$. Let $a_{1}, \ldots, a_{d}$ be a system of parameters (s.o.p.) for $R$. An $R$-module $M$ is said to be big Cohen-Macaulay $R$-module with respect to $a_{1}, \ldots, a_{d}$ if $a_{1}, \ldots, a_{d}$ is an $M$-regular sequence. Note that the definition does not require that $M$ be 
finitely generated. One of the major open problems in commutative algebra at the present time is to establish the existence of big Cohen-Macaulay modules with respect to a given s.o.p. in an arbitrary local Noetherian ring. In [7] M. Hochster has shown that if the existence of such modules could be established, then several conjectures in commutative algebra would be settled. Moreover, Hochster has established the existence of such big Cohen-Macaulay modules whenever the local ring $R$ contains a field as a subring, or has dimension not exceeding 2 (see $[7$, Ch. 4, 5] and [6]). An $R$-module $M$ for which every s.o.p. for $R$ is an $M$-regular sequence is called a balanced big Cohen-Macaulay $R$ module. Hochster has shown in $[8, \S 5]$ that such modules exist whenever $R$ contains a field as a subring. Note that every finitely generated balanced big Cohen-Macaulay R-module is maximal Cohen-Macaulay. The existence of such finitely generated modules over complete Noetherian local rings is an open problem. In Section 3 we present some equivalent conditions for the existence of maximal Cohen-Macaulay modules over local Noetherian rings of dimension 2. Also we show that over an arbitrary local Noetherian ring of dimension 2 there exists a minimax balanced big Cohen-Macaulay module. Recall that a module is called minimax module, when it has a finitely generated submodule such that the quotient by it is an Artinian module [12].

Throughout this paper, $R$ will always be a commutative Noetherian ring with non-zero identity and $I$ will be an ideal of $R$. Note that for an $R$-module $M$, the $i^{\text {th }}$ local cohomology module of $M$ with support in $V(I)$ is defined as:

$$
\mathrm{H}_{\mathrm{I}}^{\mathrm{i}}(\mathrm{M})=\underset{\mathrm{n} \geq 1}{\lim } \operatorname{Ext}_{\mathrm{R}}^{\mathrm{i}}\left(\mathrm{R} / \mathrm{I}^{\mathrm{n}}, \mathrm{M}\right) \text {. }
$$

We refer the reader to [3] for more details about local cohomology. Recall that, for each $R$-module $M$, all integers $j \geq 0$ and all prime ideals $\mathfrak{p}$ of $R$, the $j^{\text {th }}$ Bass number of $M$ with respect to $\mathfrak{p}$ is defined as $\mu_{R}^{j}(\mathfrak{p}, M)=$ $\operatorname{dim}_{k(\mathfrak{p})} \operatorname{Ext}_{R_{\mathfrak{p}}}^{j}\left(k(\mathfrak{p}), M_{\mathfrak{p}}\right)$, where $k(\mathfrak{p}):=R_{\mathfrak{p}} / \mathfrak{p} R_{\mathfrak{p}}$. Also, For an Artinian $R$ module $A$ we denote by $\operatorname{Att}_{R}(A)$ the set of attached prime ideals of $A$. For any ideal $\mathfrak{a}$ of $R$, we denote $\{\mathfrak{p} \in \operatorname{Spec} R: \mathfrak{p} \supseteq \mathfrak{a}\}$ by $V(\mathfrak{a})$. We denote the support of each $R$-module $M$ by $\operatorname{Supp}(M)$. Also, for each $R$-module $M$ we denote by $\operatorname{Ass}_{R}(M)\left(\operatorname{resp} . \operatorname{Assh}_{R}(M)\right)$ the set of associated prime ideals of $M$ (resp. the set $\left.\left\{\mathfrak{p} \in \operatorname{Ass}_{R}(M): \operatorname{dim}(R / \mathfrak{p})=\operatorname{dim}(M)\right\}\right)$. Moreover, for each $R$-module $M$, we denote by $\operatorname{Ann}_{R}(M)$ the annihilator of $M$ in $R$. Finally, for each $R$-module $M$, we denote by $\mathrm{E}_{R}(M)$ the injective envelope (or injective hull) of $M$. For any other unexplained notation and terminology we refer the reader to [3] and $[9]$.

\section{A characterization of Cohen-Macaulay modules}

In this section we shall prove some results concerning the Cohen-Macaulay modules. The main goals of this section are Theorems 2.3, 2.4 and 2.7. The following lemma, which is a generalization of [4, Proposition 1.4], is needed in the proof of Lemma 2.2. 
Lemma 2.1. Let $R$ be a commutative ring, $I$ an ideal of $R, M$ a non-zero $R$-module, $a_{1}, \ldots, a_{n} \in R$ and $J=\left(a_{1}, \ldots, a_{n}\right)$. If $I \subseteq J$ and $a_{1}, \ldots, a_{n}$ is an $M / I M$-quasi-regular sequence, then $a_{1}, \ldots, a_{n}$ is an $M$-quasi-regular sequence.

Proof. It follows easily from the definition that $J M \neq M$. Let $P\left(X_{1}, \ldots, X_{n}\right) \in$ $M\left[X_{1}, \ldots, X_{n}\right]$ be homogeneous of degree $k$ such that $P\left(a_{1}, \ldots, a_{n}\right) \in J^{k+1} M$. Then $\bar{P}\left(X_{1}, \ldots, X_{n}\right) \in M / I M\left[X_{1}, \ldots, X_{n}\right]$ is homogeneous of degree $k$ and $\bar{P}\left(a_{1}, \ldots, a_{n}\right) \in J^{k+1}(M / I M)$, where $\bar{P}=\varphi(P)$, with $\varphi$ is the canonical epimorphism. So, since $a_{1}, \ldots, a_{n}$ is a $M / I M$-quasi-regular sequence and $I \subseteq J$, it follows that the coefficients of $\bar{P}$ are in $J(M / I M)$, and hence the coefficients of $P$ are in $J M$, as required.

The following lemma is crucial in the proof of Theorem 2.3.

Lemma 2.2. Let $(R, \mathfrak{m})$ be a Noetherian local ring and $M \neq 0$ be a finitely generated $R$-module of dimension $d \geq 1$. Let $x_{1}, \ldots, x_{d} \in \mathfrak{m}$ be a system of parameters for $M$ and $I$ be an ideal of $R$ such that $I \subseteq\left(x_{1}, \ldots, x_{d}\right)$. If $\operatorname{dim}(M / I M)=$ $d$ and $M / I M$ is a Cohen-Macaulay $R$-module, then $I \subseteq \operatorname{Ann}_{R}(M)$.

Proof. Since $\operatorname{dim}(M / I M)=d=\operatorname{dim}(M)$ and $x_{1}, \ldots, x_{d}$ is a system of parameters for $M$, it is easy to see that $x_{1}, \ldots, x_{d}$ is a system of parameters for $M / I M$. Therefore, as $M / I M$ is Cohen-Macaulay it follows that $x_{1}, \ldots, x_{d}$ is an $M / I M$-regular sequence. Hence in view of [9, Theorem 16.2], it follows that $x_{1}, \ldots, x_{d}$ is an $M / I M$-quasi-regular sequence. Now since $M / I M \cong(M / \mathfrak{m} I M) /(I M / \mathfrak{m} I M)$, it follows from Lemma 2.1 that $x_{1}, \ldots, x_{d}$ is an $M / \mathfrak{m} I M$-quasi regular sequence. Whence, according to $[9$, Theorem 16.3], it follows that $x_{1}, \ldots, x_{d}$ is an $M / \mathfrak{m} I M$-regular sequence. Since $d \geq 1$, it follows from the definition that $\mathfrak{m} \notin \operatorname{Ass}_{R}(M / \mathfrak{m} I M)$. Therefore, we can deduce that $(0: M / \mathfrak{m} I M \mathfrak{m})=0$. But it is easy to see that $I M / \mathfrak{m} I M \subseteq\left(0:_{M / \mathfrak{m} I M} \mathfrak{m}\right)$. Thus, $I M / \mathfrak{m} I M=0$ and so by NAK Lemma we have $I M=0$. Which means $I \subseteq \operatorname{Ann}_{R}(M)$, as required.

The following theorem is the first main result of this section.

Theorem 2.3. Let $(R, \mathfrak{m})$ be a Noetherian local ring of dimension $d \geq 1$ and

$$
I_{1} \subset I_{2} \subset \cdots \subset I_{n-1} \subset I_{n}
$$

be a chain of ideals of $R$ such that for each $1 \leq k \leq n$, the $R$-module $R / I_{k}$ is maximal Cohen-Macaulay. Then for each system of parameters $x_{1}, \ldots, x_{d}$ of $R$, we have

$$
n \leq \ell_{R}\left(R /\left(x_{1}, \ldots, x_{d}\right)\right),
$$

where $\ell_{R}\left(R /\left(x_{1}, \ldots, x_{d}\right)\right)$ denotes the length of the $R$-module $R /\left(x_{1}, \ldots, x_{d}\right)$.

Proof. Let $2 \leq k \leq n$. If $I_{k} \subseteq\left(x_{1}, \ldots, x_{d}\right)+I_{k-1}$, then as $x_{1}+I_{k-1}, \ldots, x_{d}+$ $I_{k-1}$ is a system of parameters for local ring $R / I_{k-1}$ and by hypothesis the ring $R / I_{k} \cong\left(R / I_{k-1}\right) /\left(I_{k} / I_{k-1}\right)$ is Cohen-Macaulay, it follows from Lemma 
2.2 that $I_{k} \subseteq \operatorname{Ann}_{R}\left(R / I_{k-1}\right)=I_{k-1}$, which is a contradiction. Therefore, $I_{k} \nsubseteq\left(x_{1}, \ldots, x_{d}\right)+I_{k-1}$ and hence

$$
\left(x_{1}, \ldots, x_{d}\right)+I_{k} \nsubseteq\left(x_{1}, \ldots, x_{d}\right)+I_{k-1} .
$$

Consequently, $\ell_{R}\left(\left(\left(x_{1}, \ldots, x_{d}\right)+I_{k}\right) /\left(\left(x_{1}, \ldots, x_{d}\right)+I_{k-1}\right)\right) \geq 1$. So, we have

$$
\begin{aligned}
\ell_{R}\left(R /\left(x_{1}, \ldots, x_{d}\right)\right) & \geq \ell_{R}\left(R /\left(I_{1}+\left(x_{1}, \ldots, x_{d}\right)\right)\right) \\
& \geq 1+\ell_{R}\left(\left(I_{n}+\left(x_{1}, \ldots, x_{d}\right) /\left(I_{1}+\left(x_{1}, \ldots, x_{d}\right)\right)\right.\right. \\
& =1+\sum_{k=2}^{n} \ell_{R}\left(\left(\left(x_{1}, \ldots, x_{d}\right)+I_{k}\right) /\left(\left(x_{1}, \ldots, x_{d}\right)+I_{k-1}\right)\right) \\
& \geq 1+n-1=n .
\end{aligned}
$$

This completes the proof.

The following theorem is another application of Lemma 2.2.

Theorem 2.4. Let $(R, \mathfrak{m}, k)$ be a Noetherian local ring of dimension $d \geq 1$ and $I$ be an ideal of $R$ such that $\operatorname{dim}(R / I)=n \geq 1$. Let $x_{1}, \ldots, x_{n}$ be a system of parameters for $R / I$. If $R / I$ is a Cohen-Macaulay module and the ideal $Q:=I+R x_{1}+\cdots+R x_{n}$ is a parameter ideal of $R$, i.e., $Q$ is generated by a system of parameters for $R$, then there are elements $a_{1}, \ldots, a_{d-n} \in I$ such that $I=R a_{1}+\cdots+R a_{d-n}$ and $x_{1}, \ldots, x_{n}, a_{1}, \ldots, a_{d-n}$ is a system of parameters for $R$. In particular, if $R$ is Cohen-Macaulay, then $I$ is generated by an $R$-sequence.

Proof. By definition we have

$$
\operatorname{dim}_{k}(Q / \mathfrak{m} Q)=d .
$$

Therefore, as $\operatorname{dim}_{k}(Q / I) / \mathfrak{m}(Q / I)=\operatorname{dim}_{k}(Q / \mathfrak{m} Q+I)=n$ it follows from the exact sequence

$$
0 \rightarrow(I+\mathfrak{m} Q) / \mathfrak{m} Q \rightarrow Q / \mathfrak{m} Q \rightarrow Q /(I+\mathfrak{m} Q) \rightarrow 0
$$

that $\operatorname{dim}_{k}((I+\mathfrak{m} Q) / \mathfrak{m} Q)=d-n$. Therefore, there are elements $a_{1}, \ldots, a_{d-n}$ in $I$ such that

$$
(I+\mathfrak{m} Q) / \mathfrak{m} Q=\left(R a_{1}+\cdots+R a_{d-n}+\mathfrak{m} Q\right) / \mathfrak{m} Q .
$$

Now we have

$$
Q /(I+\mathfrak{m} Q)=\left(x_{1}+(I+\mathfrak{m} Q), \ldots, x_{n}+(I+\mathfrak{m} Q)\right),
$$

and

$$
I+\mathfrak{m} Q=R a_{1}+\cdots+R a_{d-n}+\mathfrak{m} Q,
$$

which implies $Q=R a_{1}+\cdots+R a_{d-n}+R x_{1}+\cdots+R x_{n}+\mathfrak{m} Q$. So by NAK lemma it follows that $Q=R a_{1}+\cdots+R a_{d-n}+R x_{1}+\cdots+R x_{n}$. Whence, we can deduce that $a_{1}, \ldots, a_{d-n} \in I$ is a part of a system of parameters for $R$ so $\operatorname{dim}\left(R /\left(R a_{1}+\cdots+R a_{d-n}\right)\right)=d-(d-n)=n=\operatorname{dim}(R / I)$. Now it follows from Lemma 2.2 that $I \subseteq\left(R a_{1}+\cdots+R a_{d-n}\right)$, and therefore $I=R a_{1}+\cdots+R a_{d-n}$, as required. 
The following lemma and its corollary play key role in the proof of second main result of this section.

Lemma 2.5. Let $(R, \mathfrak{m})$ be a Noetherian local ring and $M \neq 0$ be a finitely generated $R$-module of dimension $d \geq 1$. Let $I$ be an ideal of $R$ such that $I \nsubseteq \operatorname{Rad}\left(\operatorname{Ann}_{R}(M)\right)$ and $\operatorname{dim}(M / I M)=d$. Then there exists a positive integer $t$ such that the following statements hold:

(i) $\operatorname{dim}\left(I^{n} M / I^{n+1} M\right)<d$ for all integers $n \geq t$.

(ii) $\operatorname{Ass}_{R}\left(M / I^{n} M\right) \neq \operatorname{Assh}_{R}\left(M / I^{n} M\right)$ for all integers $n>t$.

(iii) The R-module $M / I^{n} M$ is not Cohen-Macaulay for all integers $n>t$.

Proof. (i) Let $\operatorname{Assh}_{R}(M)=\left\{\mathfrak{q}_{1}, \ldots, \mathfrak{q}_{k}\right\}$. Then for each $1 \leq j \leq k$ the $R_{\mathfrak{q}_{j}}$ module $M_{\mathfrak{q}_{j}}$ has finite length. Let $t:=\max \left\{\ell_{R_{\mathfrak{q}_{1}}}\left(M_{\mathfrak{q}_{1}}\right), \ldots, \ell_{R_{\mathfrak{q}_{k}}}\left(M_{\mathfrak{q}_{k}}\right)\right\}$. Then we claim that $\operatorname{Supp}_{R}\left(I^{n} M / I^{n+1} M\right) \cap \operatorname{Assh}_{R}(M)=\emptyset$ for all integers $n \geq t$. Suppose that the contrary be true. Then there is $1 \leq j \leq k$ such that $\mathfrak{q}_{j} \in$ $\operatorname{Supp}_{R}\left(I^{n} M / I^{n+1} M\right)$. So by definition we have $I^{n} M_{\mathfrak{q}_{j}} \neq I^{n+1} M_{\mathfrak{q}_{j}}$. Therefore,

$$
\begin{aligned}
t & \geq \ell_{R_{\mathfrak{q}_{j}}}\left(M_{\mathfrak{q}_{j}}\right) \geq \ell_{R_{\mathfrak{q}_{j}}}\left(M_{\mathfrak{q}_{j}} / I^{n+1} M_{\mathfrak{q}_{j}}\right) \\
& =\ell_{R_{\mathfrak{q}_{j}}}\left(M_{\mathfrak{q}_{j}} / I M_{\mathfrak{q}_{j}}\right)+\Sigma_{s=1}^{n} \ell_{R_{\mathfrak{q}_{j}}}\left(I^{s} M_{\mathfrak{q}_{j}} / I^{s+1} M_{\mathfrak{q}_{j}}\right) \geq n+1,
\end{aligned}
$$

and so $t \geq n+1$, which is a contradiction. Therefore,

$$
\operatorname{Supp}_{R}\left(I^{n} M / I^{n+1} M\right) \cap \operatorname{Assh}_{R}(M)=\emptyset
$$

for all integers $n \geq t$. Now it is easy to see that $\operatorname{dim}\left(I^{n} M / I^{n+1} M\right)<d$ for all integers $n \geq t$.

(ii) Since $\operatorname{Ass}_{R}\left(I^{n-1} M / I^{n} M\right) \subseteq \operatorname{Ass}_{R}\left(M / I^{n} M\right)$ and $\operatorname{dim}\left(M / I^{n} M\right)=$ $\operatorname{dim}(M / I M)=d$, the assertion follows from (i).

(iii) Follows from (ii) using [9, Theorem 17.2].

Corollary 2.6. Let $(R, \mathfrak{m})$ be a Noetherian local ring and $M \neq 0$ be a finitely generated $R$-module of dimension $d$. Then for any proper ideal $I$ of $R$ the following statements are equivalent:

(i) $M$ is Cohen-Macaulay and $I \subseteq \operatorname{Rad}\left(\operatorname{Ann}_{R}(M)\right)$.

(ii) $\operatorname{depth}_{R}\left(M / I^{n} M\right)=d$ for all $n \gg 0$.

Proof. The assertion follows from Lemma 2.5.

Now we are ready to give a characterization of Cohen-Macaulay modules over Noetherian local rings, which is the second main result of this section.

Theorem 2.7. Let $(R, \mathfrak{m})$ be a Noetherian local ring and $M$ be a non-zero finitely generated $R$-module of dimension $d$. Then the following conditions are equivalent:

(i) $M$ is Cohen-Macaulay.

(ii) There exists a proper ideal $I$ of $R$ such that $\operatorname{depth}_{R}\left(M / I^{n} M\right)=d$ for all $n \gg 0$.

Proof. The assertion follows from Corollary 2.6. 


\section{Cohen-Macaulay modules over Noetherian local rings of small dimension}

In this section we study the existence of Cohen-Macaulay and balanced big Cohen-Macaulay modules over Noetherian local ring of dimension 2. We shall use the following lemma in the proof of some results of this section.

Proposition 3.1. Let $(R, \mathfrak{m})$ be a Noetherian local domain of dimension $d$ and $M$ be a finitely generated $R$-module such that $\operatorname{Ass}_{R}(M)=\{0\}$ and $\mu_{R}^{0}(0, M)=$ $n \geq 1$. Then there exists an exact sequence

$$
0 \rightarrow M \rightarrow \oplus_{i=1}^{n} R \text {. }
$$

Proof. First let $d=0$. Then by hypothesis $R$ is a field and so $M$ is a vector space over $R$ of dimension of $n$ and hence $M \cong \oplus_{i=1}^{n} R$. Now let $d \geq 1$. By definition it is easy to see that there exists an exact sequence

$$
0 \rightarrow \mathrm{E}_{R}(M) \rightarrow \oplus_{i=1}^{n} \mathrm{E}_{R}(R) .
$$

Hence by assumption $F:=\oplus_{i=1}^{n} R$ we can assume that, $M$ is a finitely generated submodule of $\mathrm{E}_{R}(F)$.

Let

$$
0 \rightarrow F \rightarrow E \rightarrow E_{1} \rightarrow \cdots
$$

be a minimal injective resolution for $F$. Then in view of the definition we have $\mathrm{E}_{R}(E / F)=E_{1}$ and so $\operatorname{Ass}_{R}(E / F)=\operatorname{Ass}_{R}\left(E_{1}\right)$. But $\mathfrak{p} \in \operatorname{Ass}_{R}\left(E_{1}\right)$ if and only if $\mathrm{E}_{R}(R / \mathfrak{p})$ is a direct summand of $E_{1}$ if and only if $\mu_{R}^{1}(\mathfrak{p}, F) \neq 0$ if and only if $\mu_{R}^{1}(\mathfrak{p}, R) \neq 0$ (Because we have $F=\oplus_{i=1}^{n} R$ and so $\mu_{R}^{1}(\mathfrak{p}, F)=n \mu_{R}^{1}(\mathfrak{p}, R)$ ). Since $\mathfrak{q}=0$ is a prime ideal of $R$ it follows that the zero dimensional local ring $R_{\mathfrak{q}}$ is a field and hence is a Gorenstein ring. Therefore $\left.\mu_{R}^{1}(\mathfrak{q}, F)=n \mu_{R}^{1}(\mathfrak{q}, R)\right)=$ 0 . Hence height $(\mathfrak{p}) \geq 1$ for every $\mathfrak{p} \in \operatorname{Ass}_{R}(E / F)=\operatorname{Ass}_{R}\left(E_{1}\right)$.

Now if $M \subseteq F$, then there is nothing to prove. But, if $M \nsubseteq F$, then $M+F / F$ is a non-zero finitely generated submodule of $E_{1}$. Hence, for every $\mathfrak{p} \in \operatorname{Ass}_{R}(M+F / F)$, we have height $(\mathfrak{p}) \geq 1$, which $\operatorname{implies}$ height $(L) \geq 1$, where $L:=\operatorname{Ann}_{R}(M+F / F)$. Therefore, $L \neq 0$ and so there is an element $x \in L$ such that $x \neq 0$. But in this situation it is easy to see that $x$ is an $M$ regular element. (Note that by hypothesis we have $\operatorname{Ass}_{R}(M)=\{0\}$.) Therefore, $M \cong x M \subseteq F$. This completes the proof.

The following theorem, which is the first main result of this section, gives some equivalent conditions for the existence of maximal Cohen-Macaulay modules over local Noetherian rings of dimension 2.

Theorem 3.2. Let $(R, \mathfrak{m})$ be a local Noetherian ring of dimension 2 . Then the following conditions are equivalent:

(i) There exists a finitely generated $R$-module $M$ of dimension 2 such that the $R$-module $\mathrm{H}_{\mathfrak{m}}^{1}(\mathrm{M})$ is finitely generated.

(ii) There is $\mathfrak{p} \in \operatorname{Assh}_{R}(R)$ such that the $R$-module $\mathrm{H}_{\mathfrak{m}}^{1}(\mathrm{R} / \mathfrak{p})$ is finitely generated. 
(iii) There is $\mathfrak{p} \in \operatorname{Assh}_{R}(R)$ such that the $R$-module $\mathrm{D}_{\mathfrak{m}}(\mathrm{R} / \mathfrak{p})$ is a maximal Cohen-Macaulay $R$-module.

(iv) There exists $\mathfrak{p} \in \operatorname{Assh}_{R}(R)$ such that for some ideals $I \supset \mathfrak{p}$ of $R$ the $R$-module $M=I / \mathfrak{p}$ is maximal Cohen-Macaulay and $\operatorname{ara}(I / \mathfrak{p})=1$.

(v) There exists a maximal Cohen-Macaulay $R$-module.

Proof. (i) $\Rightarrow$ (ii) Let $\mathfrak{p} \in \operatorname{Assh}_{R}(M)$. Then it is easy to see that $\mathfrak{p} \in \operatorname{Assh}_{R}(R)$. As, $\mathfrak{p} \in \operatorname{Ass}_{R}(M)$, it follows from definition that there is an exact sequence

$$
0 \rightarrow R / \mathfrak{p} \rightarrow M \rightarrow T \rightarrow 0
$$

for some finitely generated $R$-module $T$. This exact sequence induces the following exact sequence

$$
\mathrm{H}_{\mathfrak{m}}^{0}(\mathrm{~T}) \rightarrow \mathrm{H}_{\mathfrak{m}}^{1}(\mathrm{R} / \mathfrak{p}) \rightarrow \mathrm{H}_{\mathfrak{m}}^{1}(\mathrm{M}) .
$$

Since by hypothesis the $R$-module $\mathrm{H}_{\mathfrak{m}}^{1}(\mathrm{M})$ is finitely generated, and the $R$ module $\mathrm{H}_{\mathfrak{m}}^{0}(\mathrm{~T})$ is finitely generated it follows that the $R$-module $\mathrm{H}_{\mathfrak{m}}^{1}(\mathrm{R} / \mathfrak{p})$ is finitely generated.

(ii) $\Rightarrow$ (i) Is clear.

(ii) $\Rightarrow$ (iii) In view of $[3$, Theorem 2.2.4], there is an exact sequence

$$
0 \rightarrow R / \mathfrak{p} \rightarrow \mathrm{D}_{\mathfrak{m}}(\mathrm{R} / \mathfrak{p}) \rightarrow \mathrm{H}_{\mathfrak{m}}^{1}(\mathrm{R} / \mathfrak{p}) \rightarrow 0,
$$

which implies that the $R$-module $\mathrm{D}_{\mathfrak{m}}(\mathrm{R} / \mathfrak{p})$ is finitely generated. $\operatorname{As}_{\operatorname{Ass}_{R}}(M)=$ $\{\mathfrak{p}\}$ we can find an element $x \in(\mathfrak{m} \backslash \mathfrak{p})$. It is clear that $x$ is an $R / \mathfrak{p}$-regular element and so the sequence $0 \rightarrow R / \mathfrak{p} \stackrel{x}{\rightarrow} R / \mathfrak{p}$, is exact. Therefore, for each $k \geq 1$, there is an exact sequence

$$
0 \rightarrow \operatorname{Hom}_{R}\left(\mathfrak{m}^{k}, R / \mathfrak{p}\right) \stackrel{x}{\rightarrow} \operatorname{Hom}_{R}\left(\mathfrak{m}^{k}, R / \mathfrak{p}\right) .
$$

By definition we have

$$
\mathrm{D}_{\mathfrak{m}}(\mathrm{M})=\underset{\mathrm{k} \geq 1}{\lim _{\mathrm{m}}} \operatorname{Hom}_{\mathrm{R}}\left(\mathfrak{m}^{\mathrm{k}}, \mathrm{R} / \mathfrak{p}\right),
$$

hence it follows that $x$ is a $D_{\mathfrak{m}}(\mathrm{R} / \mathfrak{p})$-regular element. In particular,

$$
\Gamma_{\mathfrak{m}}\left(\mathrm{D}_{\mathfrak{m}}(\mathrm{R} / \mathfrak{p})\right)=0 .
$$

Now the exact sequence $(\dagger)$ induces an exact sequence

$$
0 \rightarrow \mathrm{H}_{\mathfrak{m}}^{1}(\mathrm{R} / \mathfrak{p}) \stackrel{\mathrm{f}}{\rightarrow} \mathrm{H}_{\mathfrak{m}}^{1}(\mathrm{R} / \mathfrak{p}) \rightarrow \mathrm{H}_{\mathfrak{m}}^{1}\left(\mathrm{D}_{\mathfrak{m}}(\mathrm{R} / \mathfrak{p})\right) \rightarrow \mathrm{H}_{\mathfrak{m}}^{1}\left(\mathrm{H}_{\mathfrak{m}}^{1}(\mathrm{R} / \mathfrak{p})\right) .
$$

But, from the Grothendieck's Vanishing Theorem, [3, Theorem 6.1.2], we deduce that $\mathrm{H}_{\mathfrak{m}}^{1}\left(\mathrm{H}_{\mathfrak{m}}^{1}(\mathrm{R} / \mathfrak{p})\right)=0$. Consequently, the exact sequence (††) gives the following exact sequence:

$$
0 \rightarrow \mathrm{H}_{\mathfrak{m}}^{1}(\mathrm{R} / \mathfrak{p}) \stackrel{\mathrm{f}}{\rightarrow} \mathrm{H}_{\mathfrak{m}}^{1}(\mathrm{R} / \mathfrak{p}) \rightarrow \mathrm{H}_{\mathfrak{m}}^{1}\left(\mathrm{D}_{\mathfrak{m}}(\mathrm{R} / \mathfrak{p})\right) \rightarrow 0 .
$$

Since by hypothesis the $R$-module $\mathrm{H}_{\mathfrak{m}}^{1}(\mathrm{R} / \mathfrak{p})$ is of finite length and so is Artinian, it follows from the exact sequence $(\ddagger)$ that $f$ is an epimorphism and so $\mathrm{H}_{\mathfrak{m}}^{1}\left(\mathrm{D}_{\mathfrak{m}}(\mathrm{R} / \mathfrak{p})\right)=0$. Now using [3, Theorem 6.2.7] and the fact that 
$H_{\mathfrak{m}}^{0}\left(D_{\mathfrak{m}}(R / \mathfrak{p})\right)=0=H_{\mathfrak{m}}^{1}\left(D_{\mathfrak{m}}(R / \mathfrak{p})\right)$ it follows that the $R$-module $D_{\mathfrak{m}}(R / \mathfrak{p})$ is a maximal Cohen-Macaulay $R$-module.

(iii) $\Rightarrow$ (iv) Let $\mathfrak{p} \in \operatorname{Assh}_{R}(R)$ such that $\mathrm{D}_{\mathfrak{m}}(\mathrm{R} / \mathfrak{p})$ is a maximal CohenMacaulay $R$-module. Then the exact sequence

$$
0 \rightarrow R / \mathfrak{p} \rightarrow \mathrm{D}_{\mathfrak{m}}(\mathrm{R} / \mathfrak{p}) \rightarrow \mathrm{H}_{\mathfrak{m}}^{1}(\mathrm{R} / \mathfrak{p}) \rightarrow 0
$$

implies

$$
\mu_{R / \mathfrak{p}}^{0}\left(0, \mathrm{D}_{\mathfrak{m}}(\mathrm{R} / \mathfrak{p})\right)=\mu_{\mathrm{R} / \mathfrak{p}}^{0}(0, \mathrm{R} / \mathfrak{p})=1 .
$$

Therefore, in view of Proposition 3.1 there is an exact sequence $0 \rightarrow D_{\mathfrak{m}}(R / \mathfrak{p}) \rightarrow$ $\mathrm{R} / \mathfrak{p}$. Whence, it follows that $\mathrm{D}_{\mathfrak{m}}(\mathrm{R} / \mathfrak{p}) \cong \mathrm{I} / \mathfrak{p}$ for some ideals $I \supset \mathfrak{p}$ of $R$. Thus the $R$-module $M=I / \mathfrak{p}$ is maximal Cohen-Macaulay. Also, as $I / \mathfrak{p} \cong \mathrm{D}_{\mathfrak{m}}(\mathrm{R} / \mathfrak{p})$ it follows from the exact sequence $(\dagger)$ that $I / \mathfrak{p}$ has a submodule $(R x+\mathfrak{p}) / \mathfrak{p} \cong R / \mathfrak{p}$ such that $I /(R x+\mathfrak{p})$ has finite length. Consequently, $\mathfrak{m}^{n} I \subseteq(R x+\mathfrak{p})$ for some positive integer $n$. If $(R x+\mathfrak{p}) / \mathfrak{p}=R / \mathfrak{p}$, then there is nothing to prove. But in other cases if $\mathfrak{q}$ is a minimal prime divisor of $R x+\mathfrak{p}$, then using Principal Ideal Theorem we can deduce that height $(\mathfrak{q} / \mathfrak{p})=1$. In particular, $\mathfrak{q} \neq \mathfrak{m}$. But as $\mathfrak{m}^{n} I \subseteq(R x+\mathfrak{p}) \subseteq \mathfrak{q}$, it follows that $I \subseteq \mathfrak{q}$. Now it is easy to see that $\operatorname{Rad}(I / \mathfrak{p})=\operatorname{Rad}((R x+\mathfrak{p}) / \mathfrak{p})$. So from the definition we have $\operatorname{ara}(I / \mathfrak{p})=1$.

(iv) $\Rightarrow$ (v) Is clear.

$(\mathrm{v}) \Rightarrow(\mathrm{i})$ The assertion follows from [3, Theorem 6.2.7].

Before bringing the next Corollary, note that if $(R, \mathfrak{m})$ is a Noetherian local ring of dimension 2 and $R$ is universally catenary with all Cohen-Macaulay formal fibers, then $D_{\mathfrak{m}}(\mathrm{R} / \mathfrak{p})$ is finitely generated for all $\mathfrak{p} \in \operatorname{Assh}_{R}(R)$.

Corollary 3.3. Let $(R, \mathfrak{m})$ be a local Noetherian ring of dimension 2 . Then the following statements hold:

(i) For each finitely generated $R$-module $M$ of dimension 2 , if the $R$-module $\mathrm{H}_{\mathfrak{m}}^{1}(\mathrm{M})$ is finitely generated, then the $R$-module $\mathrm{D}_{\mathfrak{m}}\left(\mathrm{M} / \Gamma_{\mathfrak{m}}(\mathrm{M})\right)$ is a maximal Cohen-Macaulay R-module.

(ii) If $R$ is universally catenary with all Cohen-Macaulay formal fibers, then $\mathrm{D}_{\mathfrak{m}}(\mathrm{R} / \mathfrak{p})$ is a maximal Cohen-Macaulay $R$-module for all $\mathfrak{p} \in \operatorname{Assh}_{R}(R)$.

Proof. (i) As the $R$-module $\mathrm{H}_{\mathfrak{m}}^{1}\left(\mathrm{M} / \Gamma_{\mathfrak{m}}(\mathrm{M})\right) \cong \mathrm{H}_{\mathfrak{m}}^{1}(\mathrm{M})$ is finitely generated, $\mathrm{H}_{\mathfrak{m}}^{0}\left(\mathrm{M} / \Gamma_{\mathfrak{m}}(\mathrm{M})\right)=0$ and $\operatorname{dim}\left(M / \Gamma_{\mathfrak{m}}(M)\right)=2$, the assertion follows from the method used in the proof of Theorem 3.2.

(ii) Follows from (i), using [3, Theorem 2.2.4].

Proposition 3.4. Let $(R, \mathfrak{m})$ be a Cohen-Macaulay local (Noetherian) ring of dimension $d \geq 1$ and $\mathfrak{p}$ a prime ideal of $R$. Then the following statements hold:

(i) $\mathrm{H}_{\mathfrak{m}}^{1}(\mathrm{R} / \mathfrak{p})$ is not finitely generated if and only if $\operatorname{dim}(R / \mathfrak{p})=1$.

(ii) For every finitely generated $R$-module $N$ and each $\mathfrak{p} \in \operatorname{Att}_{R}\left(\mathrm{H}_{\mathfrak{m}}^{1}(\mathrm{~N})\right)$ we have $\operatorname{dim}(R / \mathfrak{p}) \leq 1$. 
Proof. (i) " $\Leftarrow "$ is clear. " $\Rightarrow$ " Since $\mathrm{H}_{\mathfrak{m}}^{1}(\mathrm{R} / \mathfrak{p})$ is not finitely generated, it follows that $\operatorname{dim} R / \mathfrak{p} \geq 1$. If $\operatorname{dim} R / \mathfrak{p} \geq 2$, then $\mathfrak{p}$ contains an $R$-regular sequence $x_{1}, \ldots, x_{n}$ of length $n=$ height $(\mathfrak{p})$. We have

$$
\operatorname{depth}\left(R /\left(x_{1}, \ldots, x_{n}\right)\right)=\operatorname{dim} R-n=\operatorname{dim} R-\operatorname{height}(\mathfrak{p})=\operatorname{dim} R / \mathfrak{p} \geq 2
$$

and so $\mathrm{H}_{\mathfrak{m}}^{1}\left(R /\left(x_{1}, \ldots, x_{n}\right)\right)=0$. Since height $\left(\left(x_{1}, \ldots, x_{n}\right)\right)=n=\operatorname{height}(\mathfrak{p})$ and $\left(x_{1}, \ldots, x_{n}\right) \subseteq \mathfrak{p}$ it follows that $\mathfrak{p}$ is a minimal prime ideal of $\left(x_{1}, \ldots, x_{n}\right)$ and hence $\mathfrak{p} \in \operatorname{Ass}_{R}\left(R /\left(x_{1}, \ldots, x_{n}\right)\right)$. Therefore, there exists an exact sequence

$$
0 \rightarrow R / \mathfrak{p} \rightarrow R /\left(x_{1}, \ldots, x_{n}\right) \rightarrow T \rightarrow 0
$$

for some finitely generated $R$-module $T$. This exact sequence implies that $\mathrm{H}_{\mathfrak{m}}^{1}(\mathrm{R} / \mathfrak{p}) \cong \mathrm{H}_{\mathfrak{m}}^{0}(T)$. Since $T$ is finitely generated, it follows that $\mathrm{H}_{\mathfrak{m}}^{1}(\mathrm{R} / \mathfrak{p})$ is finitely generated, which is a contradiction.

(ii) In view of [9, Theorem 6.4] $N$ has a prime filteration as $0=N_{0} \subset N_{1} \subset$ $\cdots \subset N_{n}=N$ of submodules such that for each $i$ we have $N_{i} / N_{i-1} \cong R / \mathfrak{p}_{i}$ with $\mathfrak{p}_{i} \in \operatorname{Spec}(\mathrm{R})$. We use induction on $n$. For $n=1$ the assertion follows from (i) and [3, Theorem 7.3.2]. Suppose that $n>1$ and the case $n-1$ is settled. Then by inductive hypothesis we have $\operatorname{dim}(R / \mathfrak{q}) \leq 1$ for each $\mathfrak{q} \in$ $\operatorname{Att}_{R}\left(\mathrm{H}_{\mathfrak{m}}^{1}\left(\mathrm{~N}_{\mathrm{n}-1}\right)\right) \cup \operatorname{Att}_{\mathrm{R}}\left(\mathrm{H}_{\mathfrak{m}}^{1}\left(\mathrm{~N}_{\mathrm{n}} / \mathrm{N}_{\mathrm{n}-1}\right)\right)$ and so $\operatorname{dim}\left(R / \operatorname{Ann}_{R}\left(\mathrm{H}_{\mathfrak{m}}^{1}\left(\mathrm{~N}_{\mathrm{n}-1}\right)\right)\right) \leq 1$ and $\operatorname{dim}\left(R / \operatorname{Ann}_{R}\left(\mathrm{H}_{\mathfrak{m}}^{1}\left(\mathrm{~N}_{\mathrm{n}} / \mathrm{N}_{\mathrm{n}-1}\right)\right)\right) \leq 1$. The exact sequence

$$
0 \rightarrow N_{n-1} \rightarrow N_{n} \rightarrow N_{n} / N_{n-1} \rightarrow 0
$$

induces the following exact sequence

$$
\mathrm{H}_{\mathfrak{m}}^{1}\left(\mathrm{~N}_{\mathrm{n}-1}\right) \stackrel{\mathrm{f}_{1}}{\rightarrow} \mathrm{H}_{\mathfrak{m}}^{1}\left(\mathrm{~N}_{\mathrm{n}-1}\right) \stackrel{\mathrm{f}_{2}}{\rightarrow} \mathrm{H}_{\mathfrak{m}}^{1}\left(\mathrm{~N}_{\mathrm{n}} / \mathrm{N}_{\mathrm{n}-1}\right) .
$$

From the exact sequence

$$
0 \rightarrow \operatorname{im}\left(f_{1}\right) \rightarrow \mathrm{H}_{\mathfrak{m}}^{1}\left(\mathrm{~N}_{\mathrm{n}-1}\right) \rightarrow \operatorname{im}\left(\mathrm{f}_{1}\right) \rightarrow 0,
$$

it follows that

$$
\operatorname{dim}\left(R / \operatorname{Ann}_{R}\left(\mathrm{H}_{\mathfrak{m}}^{1}\left(\mathrm{~N}_{\mathrm{n}}\right)\right) \leq 1 .\right.
$$

Let $J:=\operatorname{Ann}_{R}\left(\mathrm{H}_{\mathfrak{m}}^{1}\left(\mathrm{~N}_{\mathrm{n}}\right)\right)$. Then for each $\mathfrak{q} \in \operatorname{Att}_{R}\left(\mathrm{H}_{\mathfrak{m}}^{1}\left(\mathrm{~N}_{\mathrm{n}}\right)\right)$ we have $\mathfrak{q} \in V(J)$ and so $\operatorname{dim}(R / \mathfrak{q}) \leq 1$. This completes the inductive step.

Corollary 3.5. Let $(R, \mathfrak{m})$ be a local Noetherian ring of dimension 2 such that $R$ is a homomorphic image of a Cohen-Macaulay local ring. Then there exists a maximal Cohen-Macaulay $R$-module.

Proof. Let $\mathfrak{p} \in \operatorname{Assh}_{R}(R)$. Then using [3, Theorem 4.2.1] and Proposition 3.4 it follows that the $R$-module $\mathrm{H}_{\mathfrak{m}}^{1}(\mathrm{R} / \mathfrak{p})$ is finitely generated. Therefore the assertion follows from Theorem 3.2.

Corollary 3.6. Let $(R, \mathfrak{m})$ be a local Noetherian domain of dimension 2 . Then the following conditions are equivalent:

(i) The R-module $\mathrm{H}_{\mathfrak{m}}^{1}(\mathrm{R})$ is finitely generated.

(ii) There exists a maximal Cohen-Macaulay $R$-module. 
(iii) The $R$-module $M=I$ is maximal Cohen-Macaulay for some ideals $I$ of $R$ with $\operatorname{ara}(I)=1$.

Proof. Since $R$ is a domain it follows that $\operatorname{Assh}_{R}(R)=\{0\}$. Now the assertion follows from Theorem 3.2.

Remark 3.7. There exists a two-dimensional Noetherian local domain $(R, \mathfrak{m})$ which does not have a maximal Cohen-Macaulay module (see $[6, \S 1]$ and [5]). Obviously, $R$ is a catenary domain and by Corollary $3.5 R$ is not a homomorphic image of a Cohen-Macaulay local ring. This shows that in general an arbitrary catenary Noetherian local domain is not a homomorphic image of a CohenMacaulay local ring.

The following result, which is the second main result of this section, shows that over an arbitrary Noetherian local ring of dimension 2 there exists a minimax balanced big Cohen-Macaulay module. Recall that, a module is called minimax module, when it has a finitely generated submodule, such that the quotient by it is an Artinian module [12].

Theorem 3.8. Let $(R, \mathfrak{m})$ be a local Noetherian ring of dimension 2 and $I$ be an ideal of $R$ such that $\operatorname{dim}(R / I)=2$ and $\operatorname{Ass}_{R}(R / I)=\operatorname{Assh}_{R}(R / I)$. Then there exists a minimax balanced big Cohen-Macaulay $R$-module $M$ such that $\operatorname{Ann}_{R}(M)=I$.

Proof. In view of [3, Theorem 2.2.4], there is an exact sequence

$$
0 \rightarrow R / I \rightarrow \mathrm{D}_{\mathfrak{m}}(\mathrm{R} / \mathrm{I}) \rightarrow \mathrm{H}_{\mathfrak{m}}^{1}(\mathrm{R} / \mathrm{I}) \rightarrow 0
$$

which implies that the $R$-module $\mathrm{D}_{\mathfrak{m}}(\mathrm{R} / \mathrm{I})$ is minimax. Let $M:=\mathrm{D}_{\mathfrak{m}}(\mathrm{R} / \mathrm{I})$. Then it is easy to see that $\operatorname{Ann}_{R}(M)=I$. By method used in the proof of Theorem 3.2 we can deduce that $\mathrm{H}_{\mathfrak{m}}^{0}(\mathrm{M})=0=\mathrm{H}_{\mathfrak{m}}^{1}(\mathrm{M})$. Also it follows from [3, Theorem 6.1.2] that $\mathrm{H}_{\mathfrak{m}}^{2}(\mathrm{M}) \cong \mathrm{H}_{\mathfrak{m}}^{2}(\mathrm{R} / \mathrm{I})$. So by [3, Theorem 7.3.2] we have $\mathrm{H}_{\mathfrak{m}}^{2}(\mathrm{M}) \neq 0$. By [3, Exercise 6.1.9] we have $\mathrm{H}_{\mathfrak{m}}^{2}(\mathrm{M}) \cong \mathrm{H}_{\mathfrak{m}}^{2}(\mathrm{R}) \otimes_{\mathrm{R}} \mathrm{M}$. Since $\mathrm{H}_{\mathfrak{m}}^{2}(\mathrm{R})$ can be viewed as the direct limit of its finitely generated submodules and the functor $-\otimes_{R} M$ commutes with direct limits, it follows from the hypothesis $\mathrm{H}_{\mathfrak{m}}^{2}(\mathrm{R}) \otimes_{\mathrm{R}} \mathrm{M} \neq 0$ that $\mathrm{H}_{\mathfrak{m}}^{2}(\mathrm{R})$ has a finitely generated non-zero submodule $A$ such that $A \otimes_{R} M \neq 0$. Since the $R$-module $A$ is Artinian and finitely generated it follows that $A$ has finite length. Therefore, there is a positive integer $n$ such that $\mathfrak{m}^{n} A=0$. Hence there exists an exact sequence $\oplus_{i=1}^{t} R / \mathfrak{m}^{n} \rightarrow A \rightarrow 0$ for some positive integer $t$. Now the exact sequence

$$
\oplus_{i=1}^{t} M / \mathfrak{m}^{n} M \rightarrow A \otimes_{R} M \rightarrow 0
$$

implies that $M / \mathfrak{m}^{n} M \neq 0$. Whence, it follows that $\mathfrak{m} M \neq M$. On the other hand, we have $\operatorname{Ass}_{R}(R / I) \subseteq \operatorname{Ass}_{R}(M) \subseteq \operatorname{Ass}_{R}(R / I) \cup \operatorname{Ass}_{R}\left(\mathrm{H}_{\mathfrak{m}}^{1}(\mathrm{R} / \mathrm{I})\right)$ and $\Gamma_{\mathfrak{m}}(M)=0$ which implies $\mathfrak{m} \notin \operatorname{Ass}_{R}(M)$. But $\operatorname{Ass}_{R}\left(\mathrm{H}_{\mathfrak{m}}^{1}(\mathrm{R} / \mathrm{I})\right) \subseteq\{\mathfrak{m}\}$. Thus we have

$$
\operatorname{Ass}_{R}(M)=\operatorname{Ass}_{R}(R / I)=\operatorname{Assh}_{R}(R / I) \subseteq \operatorname{Assh}_{R}(R) .
$$


So $\operatorname{Ass}_{R}(M) \subseteq \operatorname{Assh}_{R}(R)$. Next, let $x_{1}, x_{2}$ be an arbitrary system of parameters for $R$. Then as $x_{1} \notin \mathfrak{p}$ for each $\mathfrak{p} \in \operatorname{Assh}_{R}(R)$ it follows from the fact $\operatorname{Ass}_{R}(M) \subseteq \operatorname{Assh}_{R}(R)$ that $x_{1}$ is a non-zerodivisor on $M$. The exact sequence

$$
0 \rightarrow M \stackrel{x_{1}}{\rightarrow} M \rightarrow M / x_{1} M \rightarrow 0
$$

induces the following exact sequence:

$$
0=\mathrm{H}_{\mathfrak{m}}^{0}(M) \rightarrow \mathrm{H}_{\mathfrak{m}}^{0}\left(M / x_{1} M\right) \rightarrow \mathrm{H}_{\mathfrak{m}}^{1}(\mathrm{M})=0,
$$

which implies that $\mathrm{H}_{\mathfrak{m}}^{0}\left(M / x_{1} M\right)$. Therefore, $\mathfrak{m} \notin \operatorname{Ass}_{R}\left(M / x_{1} M\right)$. Since $\operatorname{dim} R / x_{1} R=1$, this implies that $\operatorname{Ass}_{R}\left(M / x_{1} M\right) \subseteq \operatorname{Assh}_{R}\left(R / x_{1} R\right)$. Therefore, $x_{2}$ is a non-zerodivisor on $M / x_{1} M$. Since $\left(x_{1}, x_{2}\right) M \subseteq \mathfrak{m} M \neq M$, it follows from the definition that $x_{1}, x_{2}$ is an $M$-regular sequence and so $M$ is a minimax balanced big Cohen-Macaulay $R$-module with $\operatorname{Ann}_{R}(M)=I$.

Corollary 3.9. Let $(R, \mathfrak{m})$ be a local Noetherian ring of dimension 2. Then for each $\mathfrak{p} \in \operatorname{Assh}_{R}(R)$, there exists a minimax balanced big Cohen-Macaulay $R$-module $M$ such that $\operatorname{Ann}_{R}(M)=\mathfrak{p}$.

Proof. As $\operatorname{Ass}(R / \mathfrak{p})=\operatorname{Assh}(R / \mathfrak{p})$ and $\operatorname{dim}(R / \mathfrak{p})=2$ by Theorem 3.8 the assertion holds with $M:=\mathrm{D}_{\mathfrak{m}}(\mathrm{R} / \mathfrak{p})$.

The following result verifies the existence of the maximal Cohen-Macaulay modules with certain annihilators.

Theorem 3.10. Let $(R, \mathfrak{m})$ be a local Noetherian ring of dimension 2 which is a homomorphic image of a Gorenstein local ring. Let $I$ be an ideal of $R$ such that $\operatorname{dim}(R / I)=2$. Then the following conditions are equivalent:

(i) $\operatorname{Ass}_{R}(R / I)=\operatorname{Assh}_{R}(R / I)$.

(ii) There exists a Maximal Cohen-Macaulay $R$-module $M$ such that

$$
\operatorname{Ann}_{R}(M)=I \text {. }
$$

Proof. (i) $\Rightarrow$ (ii) In view of Theorem 3.8 the $R$-module $M:=\mathrm{D}_{\mathfrak{m}}(\mathrm{R} / \mathrm{I})$ is balanced big Cohen-Macaulay and $\operatorname{Ann}_{R}(M)=I$. So it is enough to prove that $M$ is finitely generated. To do this considering the exact sequence

$$
0 \rightarrow R / I \rightarrow \mathrm{D}_{\mathfrak{m}}(\mathrm{R} / \mathrm{I}) \rightarrow \mathrm{H}_{\mathfrak{m}}^{1}(\mathrm{R} / \mathrm{I}) \rightarrow 0,
$$

it is enough to prove that the $R$-module $\mathrm{H}_{\mathfrak{m}}^{1}(\mathrm{R} / \mathrm{I})$ is finitely generated. In view of $\left[3\right.$, Corollary 7.2.12] we must show that $\operatorname{Att}_{R}\left(\mathrm{H}_{\mathfrak{m}}^{1}(\mathrm{R} / \mathrm{I})\right) \subseteq\{\mathfrak{m}\}$. Suppose that the contrary is true. Then there is $\mathfrak{p} \in \operatorname{Att}_{R}\left(\mathrm{H}_{\mathfrak{m}}^{1}(\mathrm{R} / \mathrm{I})\right)$ such that $\mathfrak{p} \neq \mathfrak{m}$. Then by $[3$, Corollary 11.3.5] we have $\operatorname{dim}(R / \mathfrak{p})=1$. So it follows from $[3$, Theorem 11.3.2] that $\mathfrak{p} \in \operatorname{Ass}_{R}(R / I)$. Therefore, $\operatorname{Ass}_{R}(R / I) \neq \operatorname{Assh}_{R}(R / I)$, which is a contradiction.

(i) $\Rightarrow$ (ii) $\operatorname{As} \operatorname{Ann}_{R}(M)=I$ and $M$ is finitely generated it follows that there is an exact sequence

$$
0 \rightarrow R / I \rightarrow \oplus_{i=1}^{n} M
$$

for some positive integer $n$. Therefore, $\operatorname{Ass}_{R}(R / I) \subseteq \operatorname{Ass}_{R}(M) \subseteq \operatorname{Assh}_{R}(R)$. Now it is easy to see that $\operatorname{Ass}_{R}(R / I)=\operatorname{Assh}_{R}(R / I)$. 
Corollary 3.11. Let $(R, \mathfrak{m})$ be an analytically irreducible local ring of dimension 2. Then there exists a maximal Cohen-Macaulay $R$-module.

Proof. Since by definition $\widehat{R}$ is a domain it follows that $R$ is a domain. In view of Cohen's structure theorem $\widehat{R}$ is a homomorphic image of a regular local ring. Since $\widehat{R}$ is a domain it follows from Corollary 3.5 and Corollary 3.6 that the $\widehat{R}$-module $\mathrm{H}_{\mathfrak{m} \widehat{\mathrm{R}}}^{1}(\widehat{\mathrm{R}})$ is finitely generated. Since the $\widehat{R}$-module

$$
H_{\mathfrak{m}}^{1}(\mathrm{R}) \cong H_{\mathfrak{m}}^{1}(\mathrm{R}) \otimes_{\mathrm{R}} \widehat{\mathrm{R}} \cong \mathrm{H}_{\mathfrak{m} \widehat{\mathrm{R}}}^{1}(\widehat{\mathrm{R}})
$$

has finite length as an $\widehat{R}$-module, it follows that this $R$-module has finite length. Now the assertion follows from Corollary 3.6.

Acknowledgments. The author is deeply grateful to the referee for his/her careful reading of the paper and valuable suggestions. Also, he would like to thank from the Institute for Research in Fundamental Sciences (IPM), for its financial support.

\section{References}

[1] M. Brodmann, Asymptotic stability of Ass $\left(M / I^{n} M\right)$, Proc. Amer. Math. Soc. 74 (1979), no. $1,16-18$.

[2] Soc. 86 (1979), no. 1, 35-39.

[3] M. P. Brodmann and R. Y. Sharp, Local Cohomology: an algebraic introduction with geometic applications, Cambridge University Press, Cambridge, 1998.

[4] A. Cherrabi, Quasi-regular sequences and regular sequences, Comm. Algebra 39 (2011), no. 1, 184-188.

[5] D. Ferrand and M. Rayanaud, Fibres formelles d'un anneau local Noethérien, Ann. Sci. École Norm. Sup. 3 (1970), 295-311.

[6] M. Hochster, Cohen-Macaulay modules, Conference on Commutative Algebra (Univ. Kansas, Lawrence, Kan., 1972), pp. 120-152. Lecture Notes in Math., Vol. 311, Springer, Berlin, 1973.

[7] - Topics in the homological theory of modules over commutative rings, C.B.M.S. Regional Conference Series in Mathematics No. 24, Providence, R.I.: AMS, 1975.

[8] _ Big Cohen-Macaulay modules and algebras and embeddability in rings of Witt vectors, Conference on Commutative Algebra975 (Queen's Univ., Kingston, Ont., 1975), pp. 106-195. Queen's Papers on Pure and Applied Math., No. 42, Queen's Univ., Kingston, Ont., 1975.

[9] H. Matsumura, Commutative Ring Theory, Cambridge Univ. Press, Cambridge, UK, 1986.

[10] L. Melkersson, On asymptotic stability for sets of prime ideals connected with the powers of an ideal, Math. Proc. Cambridge Philos. Soc. 107 (1990), no. 2, 267-271.

[11] L. J. Ratliff, Jr., On the prime divisors of $I^{n}, n$ large, Michigan Math. J. 23 (1976), 337-352.

[12] H. Zöschinger, Minimax modules, J. Algebra 102 (1986), no. 1, 1-32. 
Faculty of Mathematical Sciences

Department of Mathematics

University of Mohaghegh Ardabili

56199-11367, ARdabIL, IraN

AND

SCHOOL OF MATHEMATICS

Institute for Research in Fundamental Sciences (IPM)

P.O. Box. 19395-5746, Tehran, Iran

E-mail address: bahmanpour.k@uma.ac.ir, bahmanpour.k@gmail.com 